week period in 4 district general hospitals within Yorkshire. The main outcome measures included symptoms prior to hospital presentation, length of hospital stay and length of symptoms; investigations including Chest $\mathrm{x}$-ray, CRP and blood culture, and the presence of symptomatic contacts or contacts with positive COVID-19 swab results.

Results Our study demonstrated that fever, cough and reduced oral intake were the three most common clinical presentations of COVID-19 in children. Our results are tabulated for presentation but to summarise our results; $71 \%$ of the children required a period of hospital admission and of these, $80 \%$ were admitted for between 1 to 3 days. The patients who required prolonged hospital admissions were in the younger age group or had underlying medical conditions. $100 \%$ of patients who had blood cultures performed had negative blood culture results. Only $25 \%$ of patients had bilateral consolidation on chest X-ray findings. All 21 of the patients in this study were discharged home and did not require admission to intensive care. The 2 patients with PIMS were transferred to a Paediatric Intensive Care (PICU) but have since been discharged home and remain under follow up. Our study is the first to describe photophobia as a presenting feature of COVID-19 in one patient, and chest pain in a second patient. Conclusions Fever, cough and reduced oral intake were the top 3 presentations of COVID-19 in the patients included within this study. Paediatric patients who required prolonged hospital admissions ( $>7$ days) were in the younger age group or had underlying medical conditions. This study is the first to describe photophobia as a presenting feature of COVID-19 in one patient, and chest pain in a second patient. Larger studies and more widespread testing are required to ascertain the true incidence in the paediatric population.

\section{AN AUDIT OF THE DIAGNOSTIC ASSESSMENT PATHWAY FOR AUTISM IN PRIMARY SCHOOL AGED CHILDREN IN GLOUCESTERSHIRE AGAINST NATIONAL AND INTERNATIONAL BEST PRACTICE}

Jothimani Thiagarajan, Josie Preston, Tanya Richardson, Kate Martin, Kate Lamb. UK

\subsection{6/bmjpo-2021-RCPCH.108}

Background Autism Spectrum disorder (ASD) is the most prevalent developmental disorder worldwide, affecting all ethnic backgrounds. The estimated global prevalence of autism is $1 \%$. Existing studies examining prevalence rates suggest lower diagnostic rate in developing countries. Lack of awareness, cultural factors, and access to appropriate professionals, play a role in this. The World Health Organisation (WHO) 'Thimphu Declaration' 2017 emphasizes the need for a whole-ofsociety and government approach to strengthen national capacities to provide effective support to people with ASD.

In the UK, the National Institute of Clinical Excellence (NICE) has recommended an evidenced based standard of assessment for the diagnosis of ASD. It requires multiple professional involvement; Paediatrician or psychiatrist, Speech and Language Therapist (SALT), Educational or Clinical Psychologist (EP). The NHS Long Term Plan 2019, however, acknowledges the deficiencies in this specialist service provision in the UK. Literature clearly highlights the benefits of early diagnosis and recognition of co-morbidity with ASD.

The RCPCH, State of Child Health report 2020 identifies variations in defining disabilities and learning difficulties and lack of clinical data recording for these groups. This adds to inequalities in health and educational provision.

Gloucestershire has no formal multi-disciplinary diagnostic pathway for the assessment of ASD in primary school aged children. No clinical data is collected routinely on outcomes for these children or their support needs.

Objectives To audit the process of autism assessment for primary school aged children against NICE guidelines.

To provide data to inform service development and commissioners in developing a NICE compliant autism assessment pathway and post-diagnostic support services

Methods Referrals to a specialist Paediatric neuro-disability clinic over a two month period in 2018 were retrospectively audited. These were manually categorised by reason for referral. For those referred for ASD assessment, clinic letters for up to one year after referral were reviewed. Data retrieved: number with final diagnosis of autism, time taken for diagnosis, frequency and type of co-morbidities and level of multidisciplinary involvement.

Results 46/199 referrals requested an autism assessment. 25 rejected due to lack of sufficient information. 21 accepted for an assessment for ASD. 6/21 given a final diagnosis of ASD. $7 / 21$ seen by all three of SALT, EP and Pediatrician, in line with NICE guidelines. 3/21 saw EP and no SALT and 3/21 saw SALT and no EP. The average time taken from first appointment to diagnosis was 8.6 months.

Co-morbidities identified in $5 / 6$ children diagnosed with ASD. This included ADHD, tics, developmental co-ordination disorder and learning difficulties. 16/21 not diagnosed with ASD were given alternative formulations; social, emotional and mental health needs being most common.

Conclusions Most primary children in Gloucestershire are not following an assessment process for autism which meets NICE and WHO standards, despite the UK being a developed country with nationalised health care. There was a high frequency of co-morbidity with ASD, within our small sample. Routine clinical data collection for children with additional needs is lacking. These systems need addressing locally and nationally to ensure children have equitable assessments and the right health and educational support

\section{CHRONIC CO-MORBIDITIES IN CHILDREN AND YOUNG PERSON WITH TYPE 1 DIABETES AND THE IMPACT ON GLYCAEMIC CONTROL}

Cinderella Menezes, Jain Neemisha, Olga Lipova. UK

\subsection{6/bmjpo-2021-RCPCH.109}

Background About 36,000 children in the UK have diabetes, $90 \%$ is type 1 . There are co-morbidities associated with it including autoimmune disorders, vascular complications, and psycho-social conditions.

Objectives

- Evaluate the prevalence of chronic co-morbidities - thyroid disease, coeliac disease and psychosocial co-morbidities in Children and Young Person with type 1 (CYPD T1) in our paediatric diabetic unit (PDU).

- Assess impact of having co-morbidities on glycaemic control.

Methods Our PDU cares for 245 CYPD under 19 years of age. We retrospectively collected and analysed data of mean HbA1c (mmol/mol) for CYPDT1 with and without co morbidities over 18-month period from 1st April 2019 to 30th 